\title{
ON THE CHARACTER OF CERTAIN TILTING MODULES
}

\author{
G. Lusztig And G. Williamson
}

1. Let $G$ be an almost simple, simply connected algebraic group over $\mathbf{k}$, an algebraically closed field of characteristic $p>1$. We fix a maximal torus $T$ of $G$; let $X=\operatorname{Hom}\left(T, \mathbf{k}^{*}\right)$ (with group operation written as + ). Let $\operatorname{Rep} G$ be the category of finite dimensional k-vector spaces with a given rational linear action of $G$ and let $\mathcal{T} G$ be the full subcategory of $\operatorname{Rep} G$ consisting of tilting modules (see [Do]). Let $X^{+}$be the set of dominant elements in $X$, defined in terms of a fixed choice of a set $R^{+}$of positive roots in the set of roots of $G$ with respect to $T$. For $V \in \operatorname{Rep} G$ and $\mu \in X$ let $n_{\mu}(V)$ be the dimension of the $\mu$-weight space of $V$. For $V \in \operatorname{Rep} G$ we set $[V]=\sum_{\mu \in X} n_{\mu}(V) e^{\mu} \in \mathbf{Z}[X]$, where $\mathbf{Z}[X]$ is the group ring of $X$ with standard basis $\left\{e^{\mu} ; \mu \in X\right\}$. (Actually, we have $[V] \in \mathbf{Z}[X]^{W}$ where $W$ is the Weyl group of $G$ with respect to $T$, viewed as a subgroup of $\operatorname{Aut}(X)$ and $\mathbf{Z}[X]^{W}$ denotes the subring of $W$-invariants.) According to [Do], there is a well defined bijection $\lambda \leftrightarrow T_{\lambda}$ between $X^{+}$and a set of representatives for the isomorphism classes of indecomposable objects of $\mathcal{T} G$ such that the following holds: $n_{\lambda}\left(T_{\lambda}\right)=1$; moreover, if $\mu \in X, n_{\mu}\left(T_{\lambda}\right) \neq 0$ then $\mu \leq \lambda$ ( $\leq$ as in no.2). We set $X^{++}=X^{+}+\rho$ where $\rho \in X^{+}$is defined by $2 \rho=\sum_{\alpha \in R^{+}} \alpha$. For $\zeta \in X^{++}$we set $S_{\zeta}=\left[T_{\zeta-\rho}\right]$,

$$
S_{\zeta}^{0}=\sum_{w \in W} \epsilon_{w} e^{w(\zeta)} / \sum_{w \in W} \epsilon_{w} e^{w(\rho)} \in \mathbf{Z}[X]^{W} .
$$

(Here $w \mapsto \epsilon_{w}$ is the sign character of $W$.) By general principles, for $\zeta \in X^{++}$we have

$$
S_{\zeta}=\sum_{\mu \in X^{++}} y_{\mu, \zeta} S_{\mu}^{0}
$$

where $y_{\mu, \zeta} \in \mathbf{N}$ is zero unless $\mu \leq \zeta$. One of the mysteries of the subject is that there is no known explicit formula (even conjecturally and even for $p \gg 0$ ) for the coefficients $y_{\mu, \zeta}$ above. This in contrast with the situation for the quantum group at $\sqrt[p]{1}$ when, for $\lambda \in X^{+}$, the analogue $T_{\lambda}^{\prime}$ of $T_{\lambda}$ and the corresponding element

G.L. supported in part by National Science Foundation grant DMS-1303060 and by a Simons Fellowship. 
$\left[T_{\lambda}^{\prime}\right] \in \mathbf{Z}[X]^{W}$ which records weight multiplicities in $T_{\lambda}^{\prime}$ are defined (see [A1]) and, setting $S_{\zeta}^{1}=\left[T_{\zeta-\rho}^{\prime}\right]$ for $\zeta \in X^{++}$, we have

$$
S_{\zeta}^{1}=\sum_{\mu \in X^{++}} x_{\mu, \zeta} S_{\mu}^{0}
$$

where $x_{\mu, \zeta} \in \mathbf{N}$ can be explicitly computed in terms of the polynomials $Q_{y, w}$ of [KL], see Soergel [S1,S2,S3] (it is zero unless $\mu \leq \zeta$ ).

Now $[\mathrm{Lu}]$ gives a method to express directly the characters of simple objects of $\operatorname{Rep} G$ (for $p \gg 0$ ) in terms of the characters of the irreducible representation of the quantum group, without the use of the Steinberg tensor product theorem. In this paper we show that a variation of the method of [Lu], combined with results of Donkin and Andersen, gives (at least conjecturally and for $p \gg 0$ ), a simple closed formula for the coefficients $y_{\mu, \zeta}$ (for a large set of $\zeta \in X^{++}$) in terms of the coefficients $x_{\mu, \zeta}$.

We thank H. H. Andersen for help with references.

2. Notation. For $\lambda, \mu \in X$ we write $\mu \leq \lambda$ if $\lambda-\mu \in \sum_{\alpha \in R^{+}} \mathbf{N} \alpha$.

Let $\left\{\check{\alpha}_{i} ; i \in I\right\}$ be the basis of $\operatorname{Hom}(X, \mathbf{Z})$ consisting of simple coroots. We have $X^{+}=\left\{\lambda \in X ; \check{\alpha}_{i}(\lambda) \in \mathbf{N} \quad \forall i \in I\right\}$. For any $\xi=\sum_{\lambda \in X} c_{\lambda} e^{\lambda} \in \mathbf{Z}[X]$ (with $\left.c_{\lambda} \in \mathbf{Z}\right)$ and any $h \geq 0$ we set $\xi^{(h)}=\sum_{\lambda \in X} c_{\lambda} e^{p^{h} \lambda} \in \mathbf{Z}[X]$. Let

$$
X_{r e d}^{+}=\left\{\lambda \in X ; \check{\alpha}_{i}(\lambda) \in[0, p-1] \quad \forall i \in I\right\} .
$$

For any $\lambda \in X^{+}$we define $\lambda^{0}, \lambda^{1}, \lambda^{2}, \ldots$ in $X_{\text {red }}^{+}$by $\lambda=\sum_{k \geq 0} p^{k} \lambda^{k}$; note that $\lambda^{k}=0$ for large $k$.

3. For $\lambda \in X^{+}, \nu \in X_{\text {red }}^{+}, \mu \in X^{+}$such that $\lambda+\rho=\nu+p(\mu+\rho)$ we have the Donkin tensor product formula, see [Do]:

$$
\left[T_{\lambda}\right]=\left[T_{\nu+(p-1) \rho}\right]\left[T_{\mu}\right]^{(1)},
$$

and its quantum analogue, see $[\mathrm{A} 1]$ :

$$
\left[T_{\lambda}^{\prime}\right]=\left[T_{\nu+(p-1) \rho}^{\prime}\right]\left(S_{\mu+\rho}^{0}\right)^{(1)} .
$$

For $k \geq 0$ let $X_{k}^{+}=\left\{\zeta \in X^{+} ; \zeta^{k} \in X^{++}, \zeta^{k+1}=\zeta^{k+2}=\cdots=0\right\}$. Note that $X_{k}^{+} \subset X^{++}$. For any $k \geq 0$ and any $\zeta \in X_{k}^{+}$we show:

$$
S_{\zeta}=S_{\zeta^{0}+p \rho} S_{\zeta^{1}+p \rho}^{(1)} \ldots S_{\zeta^{k-1}+p \rho}^{(k-1)} S_{\zeta^{k}}^{(k)}
$$

We argue by induction on $k$. For $k=0$ the result is obvious: we have $z=z^{0} \in X^{++}$ and $S_{\zeta}=S_{\zeta^{0}}$. Assume now that $k \geq 1$. We have $\zeta=\zeta^{0}+p\left(\zeta^{1}+p \zeta^{2}+\ldots\right)$ hence, by $(\mathrm{a})$ :

$$
S_{\zeta}=S_{\zeta^{0}+p \rho} S_{\zeta^{1}+p \zeta^{2}+\ldots}^{(1)}
$$


We have $\zeta^{1}+p \zeta^{2}+\cdots \in X_{k-1}^{+}$hence by the induction hypothesis we have

$$
S_{\zeta^{1}+p \zeta^{2}+\ldots}=S_{\zeta^{1}+p \rho} \ldots S_{\zeta^{k-1}+p \rho^{(k-2)}} S_{\zeta^{k}}^{(k-1)} .
$$

The result follows.

Now, using (b), we see that, if $\zeta \in X^{+}$satisfies $\zeta^{1}+p \zeta^{2}+\cdots \in X^{++}$then

$$
S_{\zeta}^{1}=S_{\zeta^{0}+p \rho}^{1}\left(S_{\zeta^{1}+p \zeta^{2}+\ldots}^{0}\right)^{(1)} \text {. }
$$

4. For any $h \geq 1$ and any $\zeta \in X^{+}$such that $\zeta^{h-1}+p \zeta^{h}+p^{2} \zeta^{h+1}+\cdots \in X^{++}$we define $S_{\zeta}^{h}$ by the inductive formula

$$
S_{\zeta}^{h}=\sum_{\mu \in X^{++}} x_{\mu, \zeta^{h-1}+p \zeta^{h}+p^{2} \zeta^{h+1}+\ldots} S_{\zeta^{0}+p \zeta^{1}+\cdots+p^{h-2} \zeta^{h-2}+p^{h-1} \mu} \cdot
$$

If $h=1$ this agrees with 0 (a) (the condition on $\zeta$ becomes $\zeta \in X^{++}$). If $h \geq 2$, then $S_{\zeta^{0}+p \zeta^{1}+\cdots+p^{h-2} \zeta^{h-2}+p^{h-1} \mu}^{h}$ is defined by induction since

$$
\zeta^{\prime}:=\zeta^{0}+p \zeta^{1}+\cdots+p^{h-2} \zeta^{h-2}+p^{h-1} \mu
$$

satisfies

$$
\zeta^{\prime h-2}+p \zeta^{\prime h-1}+p^{2} \zeta^{\prime h}+\cdots=\zeta^{h-2}+p \mu \in X^{++} .
$$

For any $h \geq 1$ and any $\zeta \in X^{+}$such that $\zeta^{h}+p \zeta^{h+1}+p^{2} \zeta^{h+2}+\cdots \in X^{++}$we show:

$$
S_{\zeta}^{h}=S_{\zeta^{0}+p \rho}^{1}\left(S_{\zeta^{1}+p \rho}^{1}\right)^{(1)} \ldots\left(S_{\zeta^{h-1}+p \rho}^{1}\right)^{(h-1)}\left(S_{\zeta^{h}+p \zeta^{h+1}+\ldots}^{0}\right)^{(h)} .
$$

Note that our assumption implies $\zeta^{h-1}+p \zeta^{h}+p^{2} \zeta^{h+1}+\cdots \in X^{++}$hence $S_{\zeta}^{h}$ is defined. We argue by induction on $h$. Assume first that $h=1$ and $\zeta^{1}+p \zeta^{2}+$ $p^{2} \zeta^{3}+\cdots \in X^{++}$. Then (b) reduces to $S_{\zeta}^{1}=S_{\zeta^{0}+p \rho}^{1}\left(S_{\zeta^{1}+p \zeta^{2}+\ldots}^{0}\right)^{(1)}$ which is known from $3(\mathrm{~d})$. Now assume that $h \geq 2$. In the right hand side of (a) we replace (using the induction hypothesis) $S_{\zeta^{0}+p \zeta^{1}+\cdots+p^{h-2} \zeta^{h-2}+p^{h-1} \mu}$ by

$$
S_{\zeta^{0}+p \rho}^{1}\left(S_{\zeta^{1}+p \rho}^{1}\right)^{(1)} \ldots\left(S_{\zeta^{h-2}+p \rho}^{1}\right)^{(h-2)}\left(S_{\mu}^{0}\right)^{(h-1)} .
$$

(Note that, if $\zeta^{\prime}$ is as above, then $\zeta^{\prime h-1}+p \zeta^{\prime h}+\cdots=\mu \in X^{++}$, hence the induction hypothesis is applicable.) Thus from (a) we obtain

$$
\begin{aligned}
& S_{\zeta}^{h} \\
& =\sum_{\mu \in X^{++}} x_{\mu, \zeta^{h-1}+p \zeta^{h}+p^{2} \zeta^{h+1}+\ldots} S_{\zeta^{0}+p \rho}^{1}\left(S_{\zeta^{1}+p \rho}^{1}\right)^{(1)} \ldots\left(S_{\zeta^{h-2}+p \rho}^{1}\right)^{(h-2)}\left(S_{\mu}^{0}\right)^{(h-1)} .
\end{aligned}
$$

It remains to show

$$
\sum_{\mu \in X^{++}} x_{\mu, \zeta^{h-1}+p \zeta^{h}+p^{2} \zeta^{h+1}+\ldots} S_{\mu}^{0}=S_{\zeta^{h-1}+p \rho}^{1}\left(S_{\zeta^{h}+p \zeta^{h+1}+\ldots}^{0}\right)^{(1)}
$$

that is,

$$
S_{\zeta^{h-1}+p \zeta^{h}+p^{2} \zeta^{h+1}+\ldots}^{1}=S_{\zeta^{h-1}+p \rho}^{1}\left(S_{\zeta^{h}+p \zeta^{h+1}+\ldots}^{0}\right)^{(1)} .
$$

This is known from $3(\mathrm{~d})$. Thus, (a) is proved. 
5. Now assume that $k \geq 1$ and $\zeta \in X_{k}^{+}$that is, $\zeta^{k} \in X^{++}, \zeta^{k+1}=\zeta^{k+2}=\cdots=0$. Then $S_{\zeta}^{k+1}$ is defined. We have

$$
S_{\zeta}^{k+1}=\sum_{\mu \in X^{++}} x_{\mu, \zeta^{k}} S_{\zeta^{0}+p \zeta^{1}+\cdots+p^{k-1} \zeta^{k-1}+p^{k} \mu}
$$

By 4(b), this becomes

$$
S_{\zeta}^{k+1}=\sum_{\mu \in X^{++}} x_{\mu, \zeta^{k}} S_{\zeta^{0}+p \rho}^{1}\left(S_{\zeta^{1}+p \rho}^{1}\right)^{(1)} \ldots\left(S_{\zeta^{k-1}+p \rho}^{1}\right)^{(k-1)}\left(S_{\mu}^{0}\right)^{(k)} .
$$

Hence, using 3(d), we have

$$
S_{\zeta}^{k+1}=S_{\zeta^{0}+p \rho}^{1}\left(S_{\zeta^{1}+p \rho}^{1}\right)^{(1)} \ldots\left(S_{\zeta^{k-1}+p \rho}^{1}\right)^{(k-1)}\left(S_{\zeta^{k}}^{1}\right)^{(k)} .
$$

6. According to $[\mathrm{A} 2,5.2(\mathrm{a})]$, for $\nu \in X_{\text {red }}^{+}$we have

$$
S_{\nu+p \rho}=S_{\nu+p \rho}^{1} \text { provided that } p \gg 0 .
$$

It is likely that for $\nu \in X_{\text {red }}^{+} \cap X^{++}$we have

$$
S_{\nu}=S_{\nu}^{1} \text { provided that } p \gg 0 \text {. }
$$

Note that (b) is a very special case of Conjecture 5.1 in [A2].

Proposition 7. Assume that $k \geq 0$ and $\zeta \in X_{k}^{+}$. Assume that $p \gg 0$ and that 6(b) holds. Then $S_{\zeta}=S_{\zeta}^{k+1}$.

If $k=0$ this is just the assumption $6(\mathrm{~b})$. Assume now that $k \geq 1$. Using 3(c) and 5(a) we see that it is enough to show

$$
\begin{aligned}
& S_{\zeta^{0}+p \rho} S_{\zeta^{1}+p \rho}^{(1)} \ldots S_{\zeta^{k-1}+p \rho}^{(k-1)} S_{\zeta^{k}}^{(k)} \\
& =S_{\zeta^{0}+p \rho}^{1}\left(S_{\zeta^{1}+p \rho}^{1}\right)^{(1)} \ldots\left(S_{\zeta^{k-1}+p \rho}^{1}\right)^{(k-1)}\left(S_{\zeta^{k}}^{1}\right)^{(k)} .
\end{aligned}
$$

This follows from the equalities $S_{\zeta^{j}+p \rho}=S_{\zeta^{j}+p \rho}^{1}$ for $j=0,1, \ldots, k-1$ (see $6(\mathrm{a})$ ) and $S_{\zeta^{k}}=S_{\zeta^{k}}^{1}$ which holds by the assumption $6(\mathrm{~b})$.

Corollary 8. In the setup of Proposition 7 we have

$$
S_{\zeta}=\sum_{\mu_{0}, \mu_{1}, \ldots, \mu_{k} \in X^{++}} x_{\mu_{k}, \zeta^{k}} x_{\mu_{k-1}, \zeta^{k-1}+p \mu_{k}} \ldots x_{\mu_{1}, \zeta^{1}+p \mu_{2}} x_{\mu_{0}, \zeta^{0}+p \mu_{1}} S_{\mu_{0}}^{0}
$$

Using 4(a) repeatedly, we have

$$
S_{\zeta}^{1}=\sum_{\mu_{0} \in X^{++}} x_{\mu_{0}, \zeta^{0}+p \zeta^{1}+p^{2} \zeta^{2}+\ldots} S_{\mu_{0}}^{0}
$$




$$
\begin{gathered}
S_{\zeta}^{2}=\sum_{\mu_{1} \in X^{++}} x_{\mu_{1}, \zeta^{1}+p \zeta^{2}+\ldots} S_{\zeta^{0}+p \mu_{1}}^{1}=\sum_{\mu_{0}, \mu_{1} \in X^{++}} x_{\mu_{1}, \zeta^{1}+p \zeta^{2}+\ldots} x_{\mu_{0}, \zeta^{0}+p \mu_{1}} S_{\mu_{0}}^{0}, \\
S_{\zeta}^{3}=\sum_{\mu_{2} \in X^{++}} x_{\mu_{2}, \zeta^{2}+p \zeta^{3}+\ldots} S_{\zeta^{0}+p \zeta^{1}+p^{2} \mu_{2}}^{2} \\
=\sum_{\mu_{0}, \mu_{1}, \mu_{2} \in X^{++}} x_{\mu_{2}, \zeta^{2}+p \zeta^{3}+\ldots} x_{\mu_{1}, \zeta^{1}+p \mu_{2}} x_{\mu_{0}, \zeta^{0}+p \mu_{1}} S_{\mu_{0}}^{0} .
\end{gathered}
$$

Continuing we get

$$
S_{\zeta}^{k+1}=\sum_{\mu_{0}, \mu_{1}, \ldots, \mu_{k} \in X^{++}} x_{\mu_{k}, \zeta^{k}} x_{\mu_{k-1}, \zeta^{k-1}+p \mu_{k}} \ldots x_{\mu_{1}, \zeta^{1}+p \mu_{2}} x_{\mu_{0}, \zeta^{0}+p \mu_{1}} S_{\mu_{0}}^{0}
$$

It remains to use Proposition 7.

9. For any $\zeta \in X^{++}$we can write $S_{\zeta}=\sum_{\mu \in X^{++}} r_{\mu, \zeta} S_{\mu}^{1}$ where $r_{\mu, \zeta} \in \mathbf{N}$.

Corollary 10. In the setup of Proposition 7 assume that $\mu \in X^{++}$satisfies $r_{\mu, \zeta} \neq$ 0 . Then $\mu=\zeta \bmod p X$.

Using Corollary 8 and $4($ a) we see that

$$
r_{\mu, \zeta}=\sum_{\mu_{1}, \ldots, \mu_{k} \in X^{++} ; \mu=\zeta^{0}+p \mu_{1}} x_{\mu_{k}, \zeta^{k}} x_{\mu_{k-1}, \zeta^{k-1}+p \mu_{k}} \ldots x_{\mu_{1}, \zeta^{1}+p \mu_{2}} .
$$

It follows that $\mu=\zeta^{0}+p \mu_{1}$ for some $\mu_{1} \in X^{++}$. Since $\zeta=\zeta^{0} \bmod p X$, it follows that $\mu=\zeta \bmod p X$. The corollary is proved.

11. In view of Corollary 10, one could hope that for any $\zeta, \mu \in X^{++}$such that $r_{\mu, \zeta} \neq 0$ we have $\mu=\zeta \bmod p X$. Unfortunately, this is contradicted by example (i) in [Je].

\section{REFERENCES}

[A1] H.H.Andersen, Tensor products of quantized tilting modules, Comm. Math. Phys. 149 (1992), 149-159.

[A2] H.H.Andersen, Tilting modules for algebraic groups, Algebraic Groups and their representations, NATO ASI series, Series C. Math.Phys.Sci., vol. 517, Kluwer Acad. Publ. Dodrecht, 1998.

[Do] S.Donkin, On tilting modules for algebraic groups, Math.Z. 212 (1993), 39-60.

[Je] J.G.Jensen, On the character of some modular indecomposable tilting modules for $\mathrm{SL}_{3}$, J.Alg. 232 (2000), 397-419.

[KL] D.Kazhdan and G.Lusztig, Schubert varieties and Poincaré duality, Proc. Symp. Pure Math., vol. 36, Amer. Math. Soc., 1980, pp. 185-203.

[Lu] G.Lusztig, On the character of certain irreducible modular representations, arXiv:1407.5346, Represent.Th. (to appear).

[S1] W.Soergel, Kazhdan-Lusztig polynomals and a combinatoric for tilting modules, Represent.Th. 1 (1997), 83-114. 
[S2] W.Soergel, Charakterformeln für Kipp Moduln über Kac-Moody Algebren, Represent.Th. 1 (1997), 115-132.

[S3] W.Soergel, Character formulas for tilting modules over quantum groups at roots of one, Current Developments in Math., 1997, Cambridge, MA, Int.Press Boston, 1999, pp. 161172.

Department of Mathematics, M.I.T., Cambridge, MA and Max Planck Institut FÜr MATHEMATIK, BonN 